\title{
Observing signatures of Cosmic Rays using high-energy gamma-ray telescopes
}

\author{
Olaf Reimer \\ Institute for Astro- and Particle Physics, University of Innsbruck \\ Technikerstraße 25/8, 6020 Innsbruck, Austria \\ \& \\ Kavli Institute for Particle Astrophysics and Cosmology, Stanford University \\ 2575 Sand Hill Road, Menlo Park, CA 94025, USA \\ E-mail: 0laf.Reimer@uibk.ac.at
}

\begin{abstract}
Detecting gamma-rays from discrete astronomical objects offers the potential to directly study sites of cosmic particle acceleration. Alternatively, the pervasive diffuse gamma-ray emission component caused by interactions of cosmic rays with interstellar gas and radiation fields carries information about the spatial distribution of cosmic particle accelerators and reveals details about the character of cosmic ray propagation in the Galaxy. Cosmic ray interactions leaving observable gamma-ray phenomena can be studied already in our solar system, but reach out to the Large Magellanic Cloud and the nearest most luminous starburst galaxies. Observations with both space- and ground-based gamma-ray telescopes complement each other when studying the signatures of cosmic ray interaction, acceleration, and propagation.
\end{abstract}

European Physical Society Europhysics Conference on High Energy Physics EPS-HEP 2009, July 16-22, 2009

Krakow , Poland 


\section{Introduction}

One of the foremost interesting aspects of contemporary gamma-ray astronomy can be seen in its capability to study the effects of cosmic ray interaction, acceleration, and propagation. Diffusive shock acceleration appears to be the principal mechanism behind the energetization of high-energy particles, with supernova remnants believed to be the much sought-after sources of galactic cosmic rays (CR). Although still lacking its ultimate proof we are presumably close to conclude the quest for the origin of CR in our Galaxy. Interactions between CR and ambient matter as well as radiation fields play a decisive role in this endeavor given that they give rise to gamma-ray production through neutral pion decay and Bremsstrahlung. In addition, CR electrons and positrons produce gamma rays by up-scattering ambient cosmic microwave background, infrared, optical, or even X-ray photons. This allows us in return to probe the phenomenology of CR interactions through signatures at the high-energy gamma-ray sky. The range of observable effects starts for an earth-orbiting detector by seeing the Earth glowing bright in the light of gamma-rays as the result of Cosmic Ray interactions with the Earth's atmosphere, continues by seeing objects in our solar neighborhood like the Moon and the Sun as sources of gamma-radiation, and is very prominently presented by the diffuse galactic gamma-ray emission. Even beyond accessing even remote parts in our Galaxy by their diffuse gamma-ray emission, recent gamma-ray observations were able to establish a prominent starforming region in the Large Magellanic Cloud as well as the two most nearby luminous starburst galaxies as sources of high-energy gamma radiation. Since direct CR measurements far from our Earth's vicinity are not possible, high-energy photon measurements are pristine messengers of CR induced emission phenomena in and even beyond our Galaxy.

\section{The Earth's Atmosphere Cosmic Ray induced gamma-ray emission}

The gamma-ray emission from the Earth is produced by cosmic-ray interactions with the Earth's atmosphere (often called the Earth's gamma-ray albedo). The vast majority of CR entering the atmosphere from above are protons and heavier nuclei, and atmospheric gamma-ray emission is the result of induced cascades of CR secondaries such as the decay of neutral pions and kaons and Bremsstrahlung of electrons and positrons. According to the kinematics of the collisions, the cross sections of these processes are peaked in the forward direction at high energies. The spectrum of albedo gamma-rays is not uniform: Whereas the spectrum of the inner part of the disk is soft (owing to the small number of secondary particles, typically neutral pions, that are back-scattered at large angles relative to the direction of the CR cascade), those CR entering the atmosphere near grazing incidence produce showers whose forward-moving gamma-radiations can penetrate the thin atmospheric layer making the limb bright when viewed from orbit. The geometric effect of shower production and absorption in the atmosphere producing a bright gamma-ray horizon was calculated almost forty years ago [1], followed by Monte Carlo calculations of gamma-ray production by CR interactions in the atmosphere [2]. The Earth's gamma-ray emission has been observed by several high-energy gamma-ray satellite missions starting as early as the 1960s, latest with the Energetic Gamma-ray Experiment 
Telescope (EGRET) aboard the Compton Gamma-Ray Observatory (CGRO). There are several reasons that render a study of the Earth's gamma-ray albedo useful with the Large Area Telescope (LAT) aboard the Fermi Gamma-ray Space Telescope: The Fermi-LAT data [3] provide the capability to study the gamma-ray emission from the Earth's atmosphere, unprecedented in energy range, resolution and statistical precision, and can be used to understand and model the interaction of cosmic rays in Earth's atmosphere and magnetic field. Data from Fermi-LAT have been used to study the gamma-ray emission produced in CR interactions with the Earth's atmosphere for over more than four orders of magnitude between $100 \mathrm{MeV}$ and $1 \mathrm{TeV}$ (Fig.1). The following effects characterize the LAT gamma-ray albedo observations: There is a bright limb at the Earth's horizon, which can be understood as generated by grazing-incidence cosmic-ray showers coming directly towards the LAT. There is a soft-spectrum nadir region dominated by gamma-rays back-scattered at large angles originating from cosmic-ray showers developing deep into the atmosphere. There exists an east-west modulation for energies below $\sim 10 \mathrm{GeV}$, caused by the deflection of primary cosmic rays in Earth's magnetic field. Since the Earth is such a bright source for the LAT, these data also provide valuable information for gamma-ray background studies and can eventually be used as a calibration source.

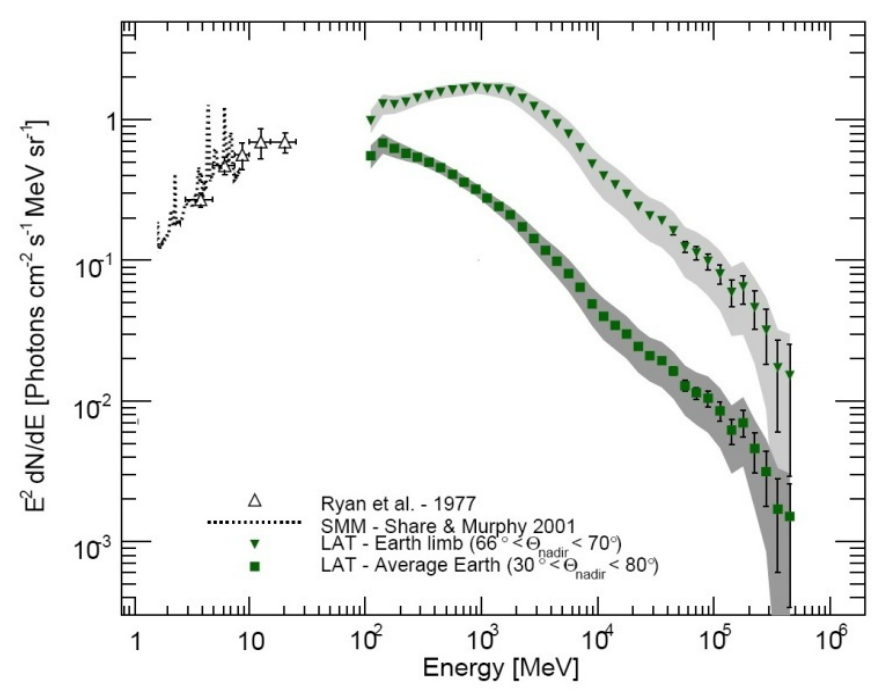

Figure 1: Spectral energy distribution of photons generated in the atmosphere of the Earth in the energy range between $1 \mathrm{MeV}$ and $1 \mathrm{TeV}$. A direct comparison of the measurements from different instruments is not straightforward, since different instruments are integrating over different regions of the Earth. Also, the measurements have been taken at different times during the solar cycle and therefore correspond to different levels of solar modulation of the primary cosmic rays. The peak of the Earth gamma-ray albedo energy flux is at $\mathrm{GeV}$ energies. The position of this peak can be understood from the power-law index of the cosmic-ray spectrum and the fact that neutral pion production is the dominant process (adapted from [3]). 


\section{Cosmic Ray interactions with the Moon's surface and the Solar disk}

Although similar physical processes relate to the gamma-ray brightness of the Moon, the observed signatures from the Earth's gamma-ray albedo, the Moon, and also the Sun are rather different. The Moon is so far the only observed gamma-ray bright solar system body with a solid surface. The Sun, however, whose gamma-ray emission is due to CR interactions with the solar atmosphere [4,5], is additionally accompanied by extended and brighter gamma-ray emission due to the inverse Compton (IC) scattering of CR electrons off solar photons [6].

Gamma-ray emission from solid solar system bodies is due to CR interactions with their surface layer material. Prominent processes involved are production and decay of neutral pions and kaons by CR nuclei, Bremsstrahlung, and Compton scattering of secondary photons. Calculations of CR interactions with the lunar surface are fairly straightforward and base on the well-measured CR spectrum and composition near Earth, and the chemical composition of the Moon rock, the regolith, which has been directly studied using probes returned to Earth by the lunar missions and by the remote sensing techniques.

EGRET, the high energy telescope aboard CGRO, discovered the energetic gamma-ray emission from the Moon [7], and also hints of the Solar disk and its halo [5], but the truly convincing measurement of the Sun was left for the Fermi observatory. The gamma-ray emission from the Moon has been confirmed by the LAT and agrees in intensity for emission models that take into account the level of solar modulation. The preliminary flux estimation for the lunar gamma-ray emission is $1.1 \pm 0.2 \times 10^{-6} \mathrm{~cm}^{-2} \mathrm{~s}^{-1}$ with a spectral index of $-3.13 \pm 0.03$ for a single power law fit in the energy range from $100 \mathrm{MeV}$ to $1 \mathrm{GeV}$ [8]. The slightly higher flux measurement is reasonably explained by a CR flux increase due the minimum activity conditions in this solar cycle. The gamma-ray emission from the Sun was measured and can be described by a power law component fit to represent the Solar disk and a model for the inverse Compton component based on the measured electron spectrum $>100 \mathrm{MeV}$ at Earth. The flux of the disk component is $\sim 3.2 \times 10^{-7} \mathrm{~cm}^{-2} \mathrm{~s}^{-1}$, while that of IC component is $\sim 1.5 \times 10^{-6} \mathrm{~cm}^{-2} \mathrm{~s}^{-1}$ within a region of $20^{\circ}$ radius centered on the Sun [9]. These flux estimates still have a substantial uncertainty primarily due to uncertainties in the IC model and the modulation of CR electrons and protons in the inner heliosphere. Nevertheless, emission from the quiet Sun is now clearly established and two different components have been separated for the first time.

\section{Cosmic Ray measurements with the gamma-ray instruments}

Primary cosmic rays can even be measured directly with ground-based gamma-ray telescopes [10]. The central idea of this method is to detect the Cherenkov light emitted by primary cosmic ray particles (the so-called Direct Cherenkov light) from the ground (Fig.2). While this component has been measured in the past by balloon experiments, the measurement from the ground can take advantage of the tremendous detection area of contemporary groundbased gamma-ray telescopes. The first application of this technique has been accomplished by the H.E.S.S. collaboration, where a measurement of the iron spectrum was presented [11]. The 
strong correlation between the Direct Cherekov light and the charge of the primary shower particle made a charge estimate possible, from which the energy dependent fraction of iron in the data was derived. The energy spectrum of iron nuclei was determined in an energy range of 13 to $200 \mathrm{TeV}$. The result confirms the flux measurements from balloon experiments with an independent technique and constitutes one of the most precise measurements in this energy range. The agreement of the distribution of the reconstructed charge $Z^{*}$ for large charges $\left(Z^{*}>\right.$ 28) disfavors a significant contribution from ultra heavy elements to the cosmic-ray flux.

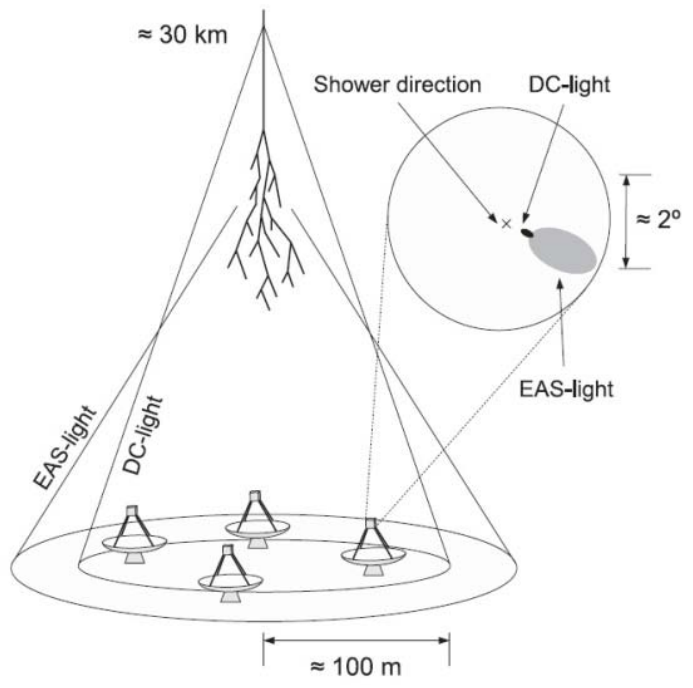

Figure 2: Schematic representation of the measurement of a cosmic-ray primary particle and the light distribution on the ground and in the camera plane using an array of imaging atmospheric Cherenkov telescopes (from [11]).

Within the last two years, both satellite and ground-based gamma-ray telescopes were able to measure the combined CR electron and positron spectrum over a unique dynamic range with unprecendented quality. Both the measurements by Fermi-LAT from $20 \mathrm{GeV}$ to $1 \mathrm{TeV}$ [12] and by the H.E.S.S. telescope array from $340 \mathrm{GeV}$ to several TeVs [13, 14] document the extraordinary capabilities of contemporary gamma-ray telescopes to measure charged cosmic rays when developing and employing non-standard analysis and rejection techniques as e.g. machine-learning algorithm. For detailled coverage of the electron measurements the reader is directly refered to the respective proceedings from this conference [15, 16].

\section{Diffuse galactic emission as the global feature of the GeV gamma-ray sky}

Gamma-rays from the interstellar medium hold great promise for studying the distribution of cosmic rays in various regions of our Galaxy. Both diffuse galactic and extragalactic gammaray emission components are of significant interest for astrophysics, particle physics, and cosmology. Once again, the observed gamma emission is understood as the product of cosmic 
ray interactions with the interstellar gas and radiation fields and thus a direct probe of CR fluxes in distant locations. It may also carry signatures of physics beyond the Standard Model of particle physics, such as information about gamma-rays originating from dark matter annihilation or decay. Diffuse galactic gamma-ray emission is a foreground for the detection and localization of point-sources and hence influences the determination of the gamma-ray observables. At the same time it is a foreground for the much fainter extragalactic gamma-ray emission component, which is the sum of contribution from unresolved sources and truly diffuse emission, including any signatures of large scale structure formation, emission produced by very-high- and ultra-high-energy CRs interacting with relic photons, and a variety of other processes [17]. Therefore, understanding the diffuse galactic gamma-ray emission is essentially a premise for such studies.

The existence of continuous gamma-ray emission was anticipated [18] and detected [19] in the very dawn of satellite-based gamma-ray astronomy. Refined measurements from the EGRET instrument aboard CGRO left one problem to be solved: The observed diffuse emission at $\mathrm{GeV}$ energies was in excess to that expected from models consistent with the directly measured CR nucleon and electron spectra [20, 21], which led to speculation that the excess emission was the long-awaited signature of dark matter annihilation [22]. More profane interpretations included variations of CR spectra in the Galaxy, contributions by unresolved point sources, and also unaccounted instrumental effects. Any model of the diffuse galactic gamma-ray emission depends on CR spectra as well as the distribution of the target gas and interstellar radiation field throughout the Galaxy. Starting from the distribution of CR sources and particle injection spectra, the propagation of CRs throughout the Galaxy is determined by taking into account relevant energy losses and gains, fragmentation and decays. In the end, CR distributions are folded with the target distributions to calculate the intensity of the diffuse gamma-ray emission components. Defining the inputs and calculating the models are far from being trivial tasks and involve data from a broad range of astronomical and astroparticle measurements [23].

Accordingly, one of the first assessments of the gamma-ray sky by the Fermi-LAT was dedicated to reinvestigate the problem of the so-called $\mathrm{GeV}$ excess from previous measurement of the diffuse gamma-ray emission. In the Galactic mid-latitude range $10^{\circ} \leq|\mathrm{b}| \leq 20^{\circ}$, the intensity scales of the Fermi-LAT-measured diffuse emission component has been found systematically softer than the previously reported spectrum. The spectral shape is compatible with that of an a priori diffuse emission model that is consistent with directly measured CR spectra. The excess emission above $1 \mathrm{GeV}$ measured by EGRET is not seen by the LAT in this region of the sky [24]. The oval agreement between the Fermi-LAT measured diffuse emission spectrum and the model (Fig. 3) shows that the fundamental radiation and loss processes are consistent with the data, thus providing a solid and conservative basis for any future work understanding the diffuse galactic emission. 

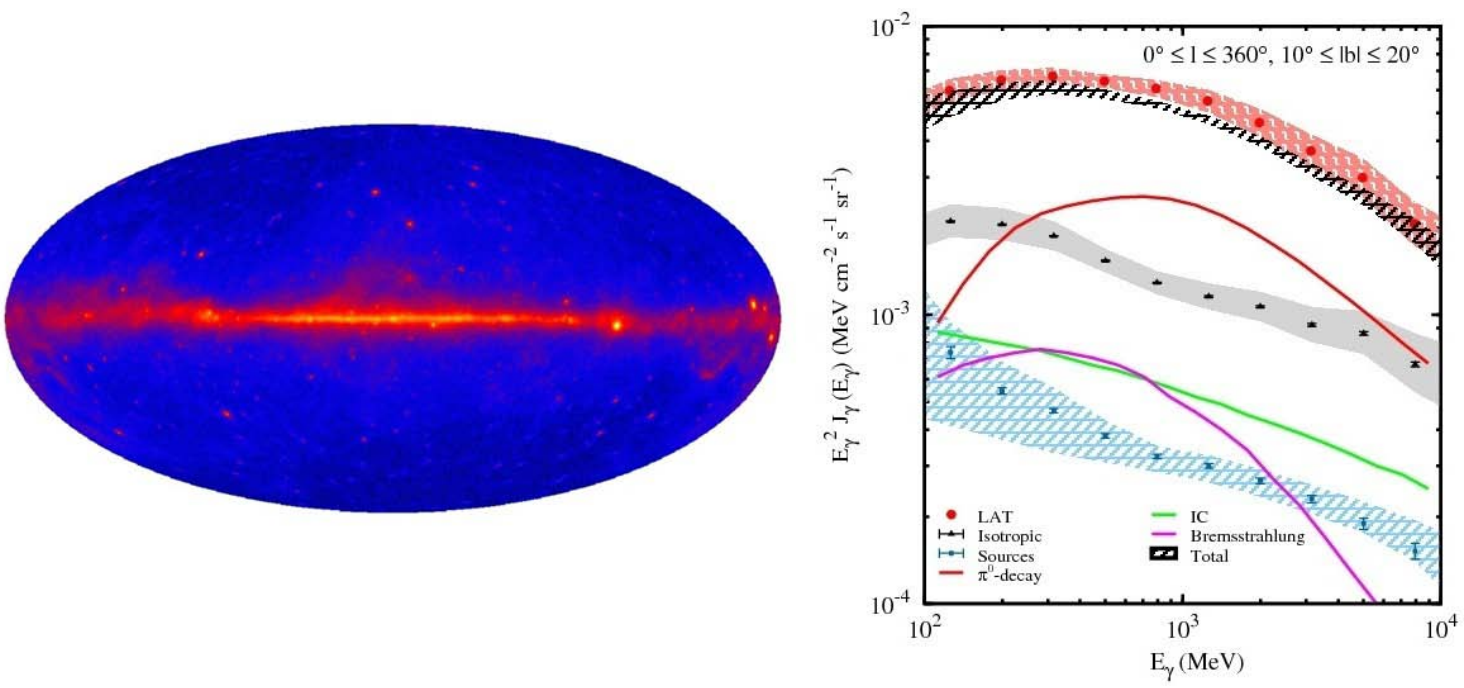

Figure 3 Left: Fermi's view of the gamma-ray sky above $300 \mathrm{MeV}$ [25]. The skymap in galactic coordinates shows the observed gamma-ray intensity. As the most prominent feature the diffuse galactic gamma-ray emission is evident. Right: Comparison of the Fermi-measured diffuse gamma-ray emission intensity averaged over all Galactic longitudes for $10^{\circ} \leq|\mathrm{b}| \leq 20^{\circ}$, and compared to an a priori model consisting of the following components: Neutral pion decay, Bremsstrahlung, inverse Compton, point-source contribution, and isotropic gamma-ray emission [24]. The overall agreement between the data and the sum of the model component is apparent.

\section{Hadronic vs. Leptonic emission process in galactic gamma-ray sources}

In the sky seen by ground-based gamma-ray telescopes, the diffuse galactic gamma-ray emission is not anymore the most prominent celestial feature. Owing to the steeply falling spectrum of the diffuse emission component above a few $\mathrm{GeV}$, the prominence of individual sources can be clearly made out at very-high-energy (VHE) gamma-rays (Fig. 4).

It has been established many years ago that the bulk of the cosmic rays measured at the Earth must originate within our Galaxy. Therefore cosmic rays with energies up to $10^{15} \mathrm{eV}$ are commonly referred to as galactic CR. Although the principal acceleration sites of galactic cosmic rays are not yet observationally established, it became clear over the course of the recent years that gamma-ray measurements offer an observational channel to identify and study cosmic ray acceleration sites in our Galaxy. Although many gamma-ray sources at $\mathrm{TeV}$ energies are meanwhile known, there are two major problems in addressing their relation to the origin of cosmic rays. The first and most basic problem is to identify gamma-ray sources with counterpart objects at other wavelengths to provide conclusive source identifications. The other challenge is to infer the nature, and spatial and energy distributions of the primary CR. Differentiating between electrons and protons as the radiating particles has proved difficult in the past, although several cases exist where one or the other is strongly favoured. 

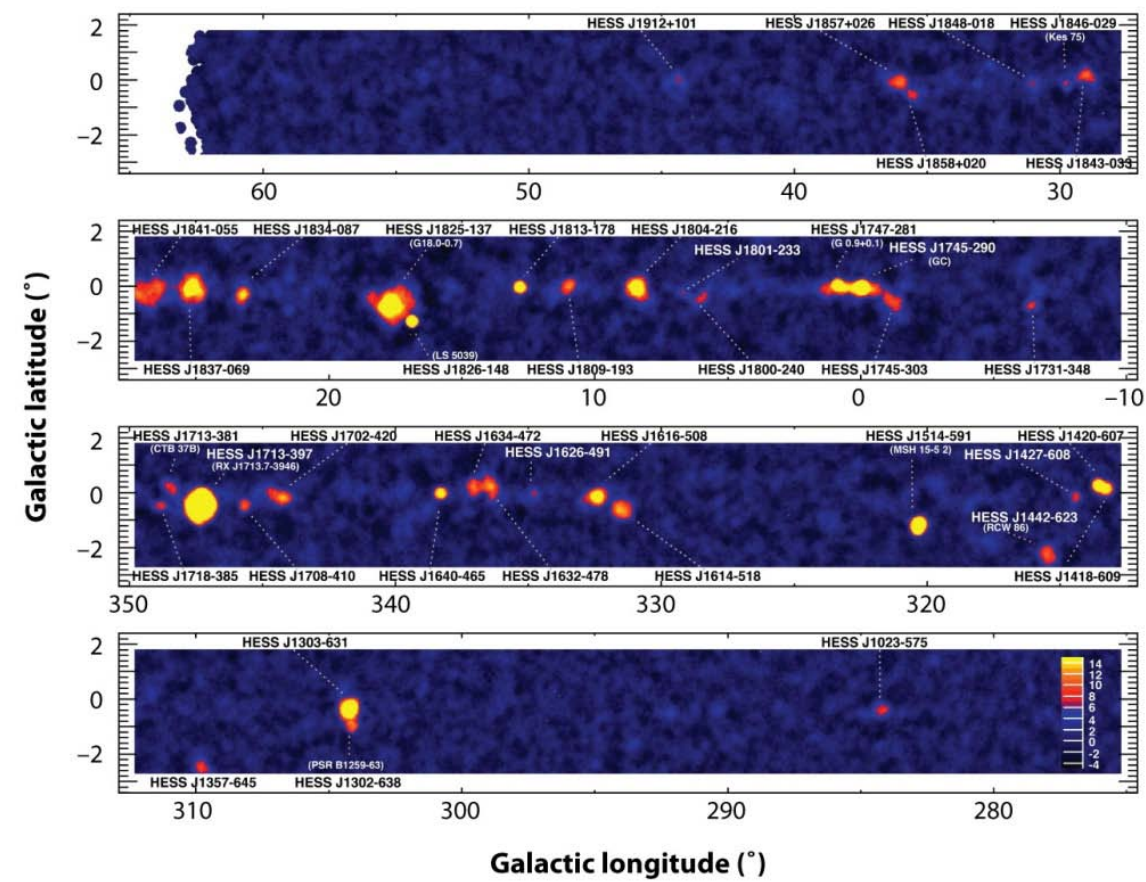

Figure 4: The H.E.S.S. survey of the inner Galaxy at VHE gamma-rays. The color scale shows the statistical significances for gamma-ray sources found. (from [26])

Whereas prominent young shell-type Supernova Remnants (SNR) (Fig. 5) have been identified through their resolved morphology, closely resembling those known from observations at (primarily) X-ray energies (e.g. RX J1713.7-3946, RX J0852.0-4622, RCW 86, SN1006), other VHE sources coincide spatially with known SNRs but cannot readily be established as such. This is the case if their angular size is in the order of the instrumental point spread function or less, leading to an inherent ambiguity in associating unresolved VHE gamma-ray sources with either the SNR or a pulsar wind nebula (PWN), powered from a compact object left from the supernova explosion. This problem cannot be addressed solely from the perspective of gamma-ray observations. Examples of such SNR-associations, now commonly referred to as composite SNR, are SNR G0.9+0.1/HESS J1747-281 (Fig. 6 left) G348.5+0.1 (CTB 37A)/HESS J1714-385, or G29.7-0.3 (Kes75)/HESS 1846-029. In a few cases, however, X-ray data argue favourable for a PWN scenario. G12.8-0.02/HESS 1813-178, or G338.3-0.0/ESS J1640-465, both unresolved at VHE energies, were subsequently targeted by XMM-Newton and Chandra, respectively. The deep X-ray observations revealed, unlike in the case of the morphological resolved SNRs, X-ray emission from a small region inside the shell of these SNRs, indicative rather for a central PWN than shell- or rim-associated SNR emission. 


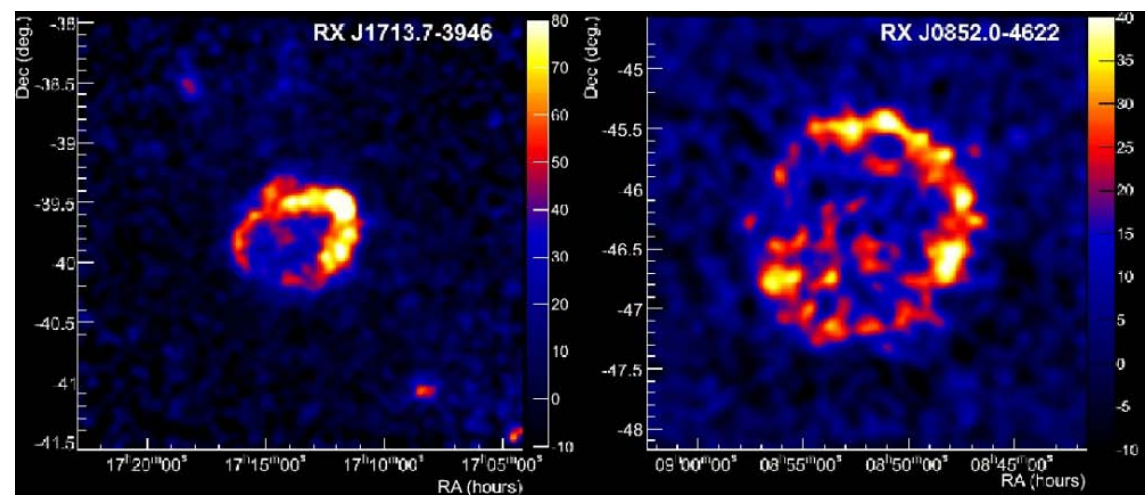

Figure 5: Morphological resolved shell-type SNRs at VHE energies: RX J1713.7-3946 and RX J0852.0-4622. The observations were made with the H.E.S.S. telescope array (adapted from [27])
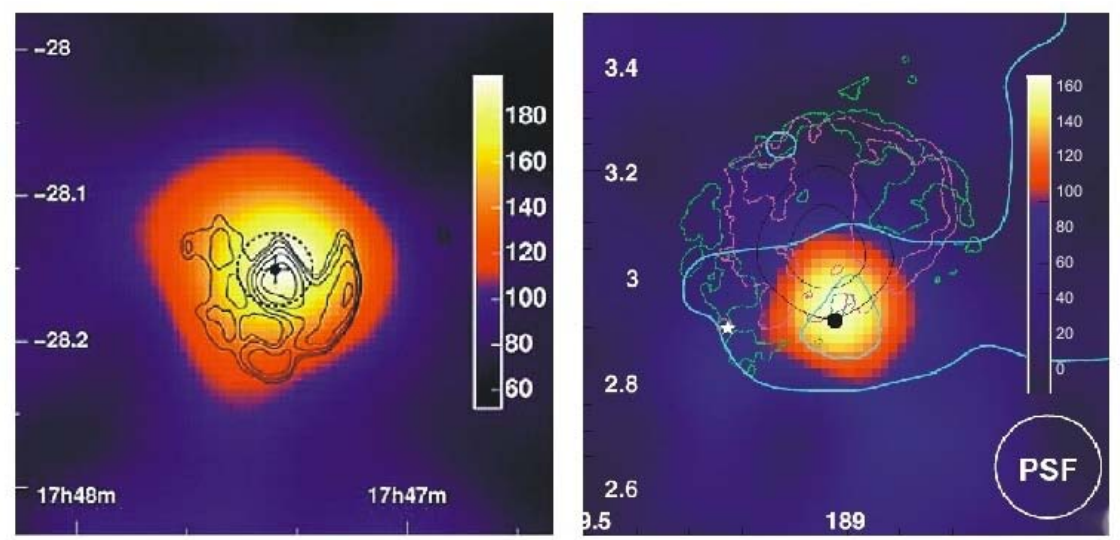

Figure 6 Left: The case of a composite VHE SNR (G0.9+0.1/HESS J1747-281). At present one cannot distinguish between resemblance of a SNR-morphology and the existence of a PWN inside an unresolved VHE gamma-ray source [28]. Right: The case of VHE gamma-ray emission seen well within the shell of a SNR (IC443/MAGIC J0616+225). The proposed scenario is a connection of VHE gamma-ray emission with overdensities of molecular material, as traced by ${ }^{12} \mathrm{CO}(\mathrm{J}=1 \rightarrow 0)$ line emission [29].

A different scenario is favoured for middle-aged SNRs like IC443 (Fig. 6 right) or W28, where gamma-ray emission is observed inside their, albeit deformed, shell structure, but in good correlation with the density distribution of molecular material. Their gamma-ray emission is interpreted as the result of interactions on the expanding SNR with vast amounts of target material (e.g. giant molecular clouds), and both density and filling factor for their target material constitute promising cases for hadronic gamma-ray emission. 


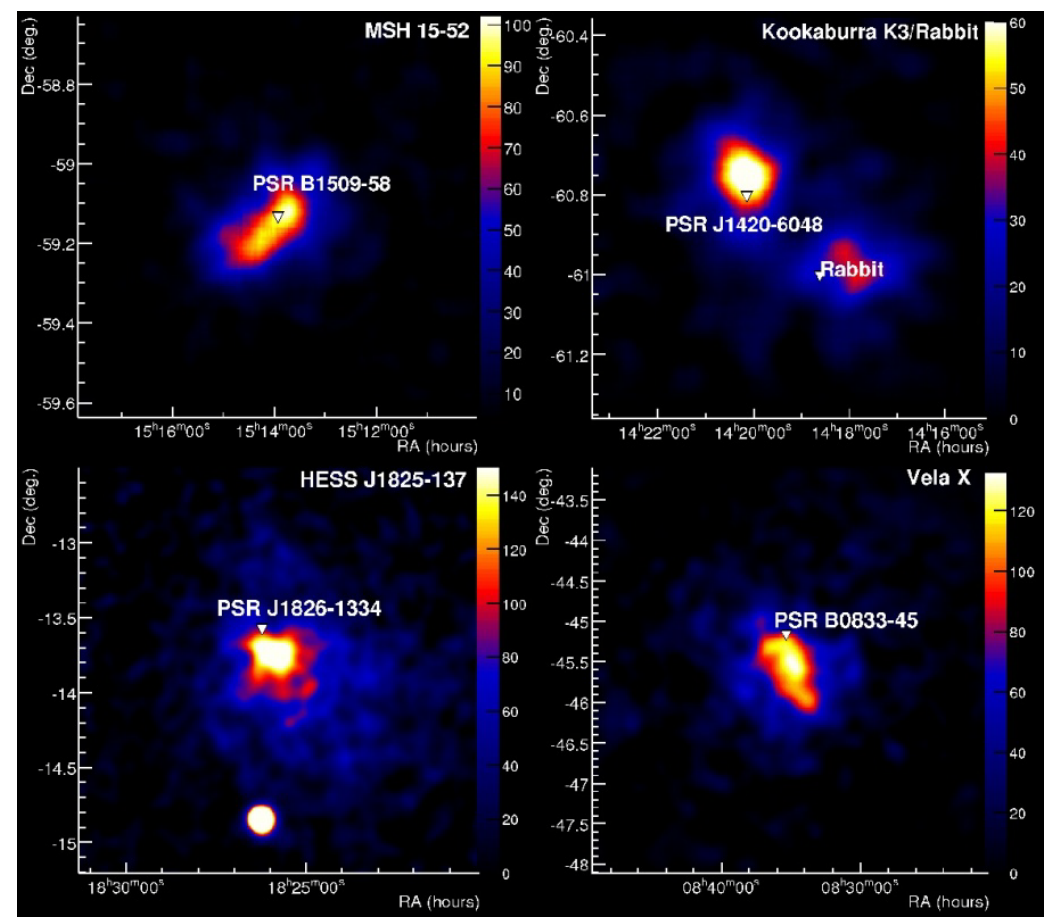

Figure 7: VHE gamma-ray smoothed excess maps of MSH 15-52 (top left), the Kookaburra region showing the emission coincident with the two non-thermal wings of the Kookaburra (top right), HESS J1825-137 (bottom left) and Vela X (bottom right). Also shown are the energetic pulsars that are thought to power the PWN. This ensemble documents the variety in the observed morphology in PWN at VHE energies (adapted from [27]).

Leaving the domain where energetic particles are confined inside an astrophysical object (as in young shell-type or composite SNRs), accelerated particles can also diffuse out into the surrounding medium. Here, the size of the emitting region itself defines the spatial extent of the gamma-ray source; consequently, a variety of different source morphologies are seen among the class of VHE pulsar wind nebulae (Fig. 7). Long since being preceded by the Crab Nebular, which establishes itself as an unique cosmic laboratory through its exceptional broad coverage over the entire electromagnetic spectrum, pulsar wind nebulae meanwhile represent the numerically dominant class of Galactic VHE objects. Their observed gamma-ray morphology is manifold: spatially extended, and often asymmetric to the celestial location of the respective pulsar believed to be powering the PWN. Asymmetric PWN have been known from X-ray observations before, but on comparably smaller scales. Given the variety among their VHE gamma-ray emission size, orientation, and center-of-gravity, in some cases the identification with a PWN remains only suggestive, but not settled. Therefore population studies start to fill in the missing pieces, e.g. by systematically probing a connection between the energetics of the powering pulsar and VHE-detected PWN. 

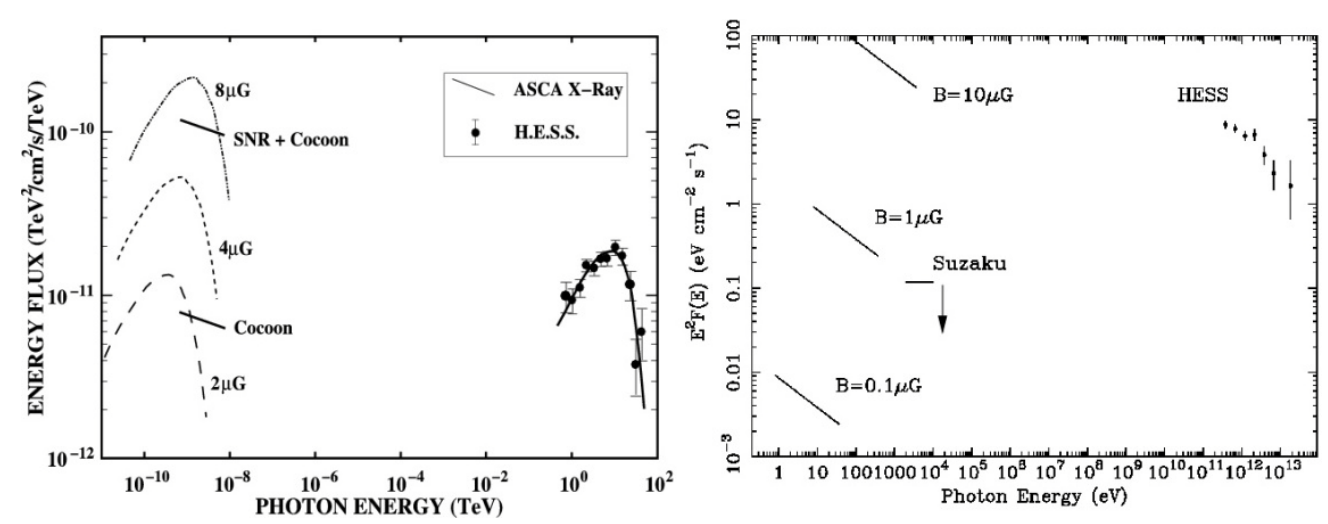

Figure 8 Left: Spectral energy distribution (SED) of the Vela X (HESS J0835-455) Pulsar Wind Nebula. The broadband fit in an inverse Compton emission scenario is shown. The predicted synchrotron flux is shown for three possible magnetic field strengths [31]. Right: SED of HESS J1616-508. The X-ray flux upper limit is well below the measured gamma-ray flux [32]. Assuming that a population of high-energy electrons is responsible for the gamma-ray emission, the expected X-ray intensity due to synchrotron radiation depends on the magnetic field in the source, as indicated. For this object an X-ray counterpart to the VHE gamma-ray source has not been found yet. Such 'Dark Accelerators' are more favourable explained by dominance of hadronic gamma-ray emission processes.

Due to the dramatic increase in the number of known $\mathrm{GeV}$ gamma-ray pulsars from observations by Fermi-LAT [30], there are often connections found between VHE PWN or unidentified VHE gamma-ray source with an energetic pulsar believed to power a related VHE emitter. In case of observed energy dependent morphology change, where spectral softening of the gamma-ray emission increasing distance to the pulsar is indicated, preference for an Inverse Compton scenario can be concluded. But even a peculiar shaped high-energy spectrum as of Vela X (HESS J0835-455, Fig. 8 left) does not offer a clear imprint of the underlying radiation processes, and both leptonic and hadronic emission scenarios are able to accommodate the data [31]. The magnetic field strength becomes the critical parameter in modelling the high-energy emission from a PWN, too. For those Galactic VHE gamma-ray sources currently lacking a convincing MWL association, the situation for X-ray counterpart searches can become desperate.

Many VHE gamma-ray sources are characterized by a typical ratio of VHE gamma-ray energy flux to X-ray energy flux in the order of unity. In case of HESS J1616-508 (Fig. 8 right) the upper limit on the X-ray flux [32] implies that at least 55 times more energy goes into gamma rays. For an electron accelerator and typical Galactic magnetic fields of about a few $\mu \mathrm{G}$, one would expect a ratio around unity, ruling effectively out an electronic origin of the gammaray emission. However, in case that synchrotron radiation is observed at energies of some $\mathrm{keV}$ their parent particle distribution is electrons at $\sim 100 \mathrm{TeV}$; yet the VHE gamma-rays are produced by lower-energy electrons in the 1 to $10 \mathrm{TeV}$ range. Thus, if the electron energy spectrum has a sharp cutoff between 10 and $100 \mathrm{TeV}$, the two data sets could be reconciled. 


\section{The role of Stellar Winds and Star Formation}

All known Galactic VHE gamma-ray sources are directly or indirectly associated with massive star formation. Both the end points of the massive stellar lifecycles, SNRs and pulsars, and massive stars with compact objects as companions appear to be $\mathrm{TeV}$ emitters. Thus it is natural to consider that massive stars can accelerate particles to $\mathrm{TeV}$ energies also in absence of a compact object. The idea of particle acceleration at shock fronts formed by expanding and colliding stellar winds exists since several decades [33, 34, 35, 36]. So far there are no unambiguous detections of individual colliding wind systems at high-energy gamma-rays, but intriguing hints exist in the observed $\mathrm{TeV}$ gamma-ray emission from the vicinity of the young stellar cluster Westerlund 2 [37], itself distinguished by containing the second largest concentration of massive young stars in our galaxy. The recent report of $\mathrm{GeV}$ gamma-ray emission spatially coincident with Eta Carina by AGILE [38] might constitute the discovery of a colliding wind binary system above $100 \mathrm{MeV}$.

Recently Fermi-LAT observations of the Large Magellanic Cloud revealed the active starburst region 30 Doradus as a bright gamma-ray source [39]. These observations provide an interesting new insight into the link between sites of CR acceleration and gamma-ray emission, and suggest that both are tightly connected. 30 Dor is known as the most active starburst region within our Local Group of galaxies, and it comes to no surprise that it is now found as the most powerful cosmic-ray acceleration site in the LMC. These findings support the concept that CR are accelerated in massive star forming regions as a result of large amounts of kinetic energy that are input by the stellar winds and supernova explosions into the interstellar medium.

The supernova remnant paradigm for the origin of galactic cosmic rays [40, 41] can also be tested by measuring gamma-ray emission from star-forming galaxies. Starburst galaxies, in particular, should have larger gamma-ray intensities compared to normal galaxies due to their increased rate of star-formation and generally greater amounts of gas and dust that reprocess light into the IR, and, with photons, serve as targets for gamma-ray production by cosmic ray electrons and ions. A sufficiently increased gamma-ray production rate will allow the detection of these phenomena [42] with the present generation of satellite- and ground-based gamma-ray instruments. Indeed, the ground-based Cherenkov telescope arrays VERITAS and H.E.S.S. have recently reported the detection of the nearest luminous starburst galaxies M82 and NGC253 as VHE gamma-ray emitter [43, 44], and the Fermi-LAT the detection of GeV emission from M82 and NGC253 [45]. The measured spectra and fluxes are compatible with predictions based on theories of cosmic-ray origin from supernovae in star-forming galaxies: Both starburst galaxies, though having less gas than the Milky Way, have two to four times greater gamma-ray luminosity, suggesting a connection between active star formation and enhanced cosmic-ray energy densities in these objects, too.

\section{Cosmic ray acceleration and storage in galaxy clusters}

Galaxy clusters and the filaments connecting them are the largest gravitational bound structures in the universe. Despite presently not being detected at high-energy gamma-rays [46], 
the following general considerations reinforce the belief that clusters of galaxies do constitute candidate high energy radiation emitters: (1) The bulk of CR accelerated within the cluster volume is confined over cosmological timescales [42, 47], thereby enhancing the possibility of inelastic proton-proton collisions and subsequent gamma ray production through neutral pion decay. (2) Large scale shocks associated with cosmological structure formation are able to accelerate electrons to $\mathrm{TeV}$ energies owing to IC scattering off the universal photon background [48]. (3) Gamma ray production in the intercluster medium can be accomplished by IC scattering from secondary electrons, from Bethe-Heitler pair production between Ultra High Energy CR and cosmic microwave background photons, and via non-thermal Bremsstrahlung. The discovery of gamma rays from clusters of galaxies, or more generally from large scale structures in the universe would represent an important step towards a better understanding of the processes of CR acceleration and confinement.

We await the results from deep Fermi-LAT observations towards galaxy clusters for a profound assessement of the observational situation and implications at high-energy gammarays.

\section{Outlook}

Connections from gamma-ray observations to cosmic rays can be made starting from our Earth's gamma-ray albedo up to nearby starburst galaxies. Over the coming years, observations carried out by the Fermi Gamma-ray Space Telescope at GeV energies and ground-based imaging Cherenkov telescope arrays H.E.S.S., MAGIC, and VERITAS at VHE energies will further improve our knowledge about cosmic ray interaction, acceleration, and propagation, to be manifest in a variety of cosmic phenomena. The planned large imaging Cherenkov telescope observatories $C T A^{1}$ and AGIS, as well as the ground-based water Cherenkov experiment HAWK will have the potential to cover the energy range from few $\mathrm{GeV}$ to hundreds of $\mathrm{TeV}$, which, in conjunction with Fermi-LAT, allow an unbroken coverage over seven orders of magnitude in gamma-ray energies. The nonthermal universe is wide open for studying the signatures of cosmic rays by means of contemporary gamma-ray astronomy.

${ }^{1}$ CTA - Cherenkov Telescope Array (http:// www.cta-observatory.org); AGIS - Advanced Gamma-Ray Imaging System (http://www.agis-observatory.org); HAWK - High Altitude Water Cherenkov Experiment (http://hawc.umd.edu) 


\section{References}

[1] F.W. Stecker, Nature 242, 59 (1973)

[2] D.J. Morris, J. Geophys. Res. 89, 10685 (1984)

[3] A.A. Abdo et al. (Fermi-LAT collaboration), Phys. Rev. D 80:122004 (2009)

[4] D. Seckel, T. Stanev, \& T.K. Gaisser, ApJ., 382, 652 (1991)

[5] E. Orlando, \& A.W. Strong, A\&A, 480, 847 (2008)

[6] I.V. Moskalenko, T.A. Porter, \& S.W. Digel, ApJ. Lett., 652, L65 (2006)

[7] D.J. Thompson, et al., J. Geophys. Res. 102, 14735 (1997)

[8] N. Giglietto for the Fermi-LAT collaboration, 2009 Fermi Symposium, eConf Proceedings C091122 [arXiv: 0912.3734$]$

[9] N. Orlando for the Fermi-LAT collaboration, 2009 Fermi Symposium, eConf Proceedings C091122 [arXiv: 0912.3775]

[10] D.B. Kieda, S.P. Swordy, \& S.P. Wakely, APh 15, 287 (2001)

[11] F.A. Aharonian et al. (H.E.S.S. collaboration), Phys. Rev. D 75:042004 (2007)

[12] A.A. Abdo et al. (Fermi-LAT collaboration), PRL 102, Issue 18:181101 (2009)

[13] F.A. Aharonian et al. (H.E.S.S. collaboration), PRL 101, Issue 26:261104 (2008)

[14] F.A. Aharonian et al. (H.E.S.S. collaboration), A\&A 508, 561 (2009)

[15] L. Tibaldo for the Fermi-LAT collaboration, Proceedings of the 2009 Europhysics Conference on High Energy Physics, PoS(EPS-HEP 2009)096

[16] K. Egberts for the H.E.S.S. collaboration, Proceedings of the 2009 Europhysics Conference on High Energy Physics, PoS(EPS-HEP 2009)097

[17] C. Dermer, Proceedings of the First GLAST Symposium, AIP Conference Proceedings, Volume 921, pp. 122-126 (2007)

[18] P. Morrison, On Gamma-Ray Astronomy, Il Nuovo Cimento 7, 858-865 (1958)

[19] G.W. Clark, G.P. Garmire \& W.L. Kraushaar, ApJ 153, L203 (1968)

[20] S.D. Hunter et al., ApJ 481, 205 (1997)

[21] A.W. Strong, I.V. Moskalenko \& O. Reimer, ApJ 537, 763 (2000)

[22] W. de Boer et al., PRL 95, Issue 20:209001 (2005)

[23] A.W. Strong, I.V. Moskalenko \& V.S. Ptuskin, ARNPS 57, 285-327 (2007)

[24] A.A. Abdo et al. (Fermi-LAT collaboration), PRL 103, Issue 25:251101 (2009)

[25] A.A. Abdo et al. (Fermi-LAT collaboration), ApJS (2010) [arXiv : 1002 . 2280]

[26] J.A. Hinton \& W. Hofmann , ARAA 47, 523-565 (2009) 
[27] S. Funk, Adv. Space Res. 41, 464 (2008)

[28] F.A. Aharonian et al. (H.E.S.S. collaboration), Science, 307, 1938 (2005)

[29] J. Albert J. et al. (MAGIC collaboration), ApJ, 664 L87 (2007)

[30] A.A. Abdo et al. (Fermi-LAT collaboration), ApJS (2010) [arXiv : 0910 .1608]

[31] F.A. Aharonian et al. (H.E.S.S. collaboration), A\&A, 448, L43 (2006)

[32] H. Matsumoto et al., PASJ, 59 199, (2006)

[33] M. Casse \& J.A. Paul, ApJ 237, 236 (1980)

[34] C.J. Cesarsky \& T. Montmerle, Space Sci. Rev. 36, 173 (1983)

[35] H.J. Völk, Space Sci. Rev. 36, 3 (1983)

[36] D. Eichler \& V. Usov, ApJ 402, 271 (1993)

[37] F.A. Aharonian et al. (H.E.S.S. collaboration), A\&A 467, 1075 (2007)

[38] M. Tavani et al., ApJ 698, L142 (2009)

[39] J. Knödelseder for the Fermi-LAT collaboration, 2009 Fermi Symposium, eConf Proceedings C091122 [arXiv: 0912.4163]

[40] V.L. Ginzburg, \& S.I. Syrovatskii, The Origin of Cosmic Rays (New York: Macmillan) (1964)

[41] S. Hayakawa, Cosmic Ray Physics (New York: Wiley-Interscience) (1969)

[42] H.J. Völk, F.A. Aharonian \& D. Breitschwerdt, Space Sci. Rev. 75, 279 (1996)

[43] V.A. Acciari et al. (VERITAS collaboration), Nature 462, 770-772 (2009)

[44] F. Acero et al. (H.E.S.S. collaboration), Science, 326 (5956), 1080 (2009)

[45] A.A. Abdo et al. (Fermi-LAT collaboration), ApJ 709 L152 (2010)

[46] O. Reimer et al., ApJ 588, 155 (2003)

[47] V.S. Berezinsky, P. Blasi \& V.S. Ptuskin, ApJ 487, 529 (1997)

[48] A. Loeb \& E. Waxman, Nature 405, 156 (2000) 